\title{
Accuracy of Surface Plate Measurements - General Purpose Software for Flatness Measurement
}

\section{Dr. ir. J. Meijer - Submitted by Prof. ir. C. J. Heuvelman (1), University of Twente/Netherlands Received on January 11, 1990}

Flatness departures of surface plates are generally obtained from straightness measurements of lines on the surface. A computer program has been developed for on-line measurement and evaluation, based on the simultaneous coupling of measurements in all grid points. Statistical methods are used to determine the accuracy of the measurements. The program runs on standard personal computers and supports different types of measuring instruments like electronic levels, autocollimators, laser interferometers or straight edge based instruments. Apart from the given height map, some meaningful characteristic parameters: sphericity, torsion and waviness are obtained. They have been proven very valuable to record long term effects of surface plates. Reliable measurements with an accuracy of $0.1 \mu \mathrm{m} / \mathrm{m}$ demonstrate the capabilities of the method.

KEYWORDS: Flatness, straightness, surface plate, measurement, accuracy

\section{Introduction}

The significance of reliable flatness measurements has already been recognized for many years, because flatness is the basis for a wide range of geometrical measurements. The need for high precision flatness standards has increased with the rise of precision engineering. Geometrical errors have crossed the one micrometer border line and the term Nanotechnology, as coined by Taniguchi, becomes more and more usual (McKeown, 1987). From this point of view the quality of technical surfaces ranks as a key technology (Trumpold, 1988). Flatness standards are also important to improve the accuracy of precision machines in combination with forecasting compensatory control stratcgies. Oya (1987) reports an $80 \%$ error reduction for a three-coordinate measuring machine. The same improvement has been found by Park (1988) from on-line cutting experiments. Error correction appears to be more effective in flatness measurement than in straightness measurement. As shown by Taniguchi (1983) there is an almost constant improvement of accuracy, more than a factor of 2 every 10 years. A target of the $24^{\text {th }}$ large scale Japanese MITI project is a shape accuracy of $0.1 \mu \mathrm{m} / \mathrm{m}$ or less, within 10 years (Anon. 1989) To realize such accuracies, the measurement function is integrated more and more as part of the production function. This paper is directed to calibration measurements of flatness standards like surface plates or tables for measuring machines. An on-line measuring system has been developed, using personal computers.

\section{Measurement methods}

Three different measurement principles for flatness measurement can be distinguished:

1. The entire surface is compared with a known reference surface (holistic methods).

2. Points on the surface are compared to a reference plane.

3. The straightness of lines in the surface is measured.

Flatness information is generated afterwards, by coupling the lines in a proper way. It has been shown (Meijer, 1989) that the holistic methods: the three plate methods, the interferometric, holographic and Moirt methods are not available for highly accurate flatness measurements of large surfaces. Also the second category of measurement methods is hardly used, mainly due to their limited accuracy. For the third method, the measurement of the straightness of lines in the surface, three different methods are available:

3a) Absolute method: Direct measurement of the distance to a straight datum line (height $b$ ).

3b) Differential method: Measurement of the tilt angles between two successive points, compared to a constant reference direction, first derivative $\frac{\mathrm{dh}}{\mathrm{dx}}$.

3c) Measurement of the position of a third point with respect to a line through two preceding points, second derivative $\frac{d^{2} h}{d x^{2}}$.

The direct (absolute) measurements can be performed by using materialized references like straight edges, stretched wires etc. or by non-materialized axes like optical axes or laser beams. For the second class of methods, instruments like autocollimators, electronic levels and laser interferometers are available. Instruments based on the third category are Spherometers, Three-point-comparators or Beam-comparators. The accuracy of the direct measurements varies, for $2 \mathrm{~m}$ long lines, from $0.5 \mu \mathrm{m}$ when using calibrated straight edges to $0.2 \mu \mathrm{m}$ when using a laser beam. The differential instruments measuring the first derivative have an ultimate accuracy of $0.1 \mu \mathrm{m}$ at $\$ 100 \mathrm{~mm}$ interval. For long lines the accuracy will be lower, due to a cumulation of errors along the line. With a given reference direction the error multiplication factor is proportional to the square root of the number of measurements. Using the regression line as a reference the trend of the cumulated errors is removed, just like the drift of the instrument with respect to a constant reference.

Simulations show that the distribution of the errors with respect to the regression line becomes approximately constant. The mean standard deviation with respect to the regression line is about $\frac{1}{4} \sigma_{n} \sqrt{n}$ where $\sigma_{m}$ is the standard deviation of the individual measurements and $n$ the number of measurements on the line. (Fig.1)

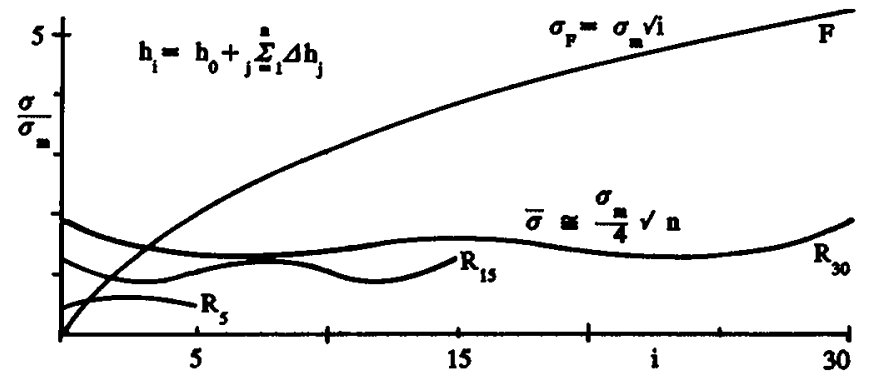

Figure 1 The standard deviation with respect to a fixed line (F) or regression lines $\left(R_{n}\right)$ with $n$ measured height differences.

With the regression line as a straightness reference the accuracy of the heights obtained from the differential measurements is in the same order as obtained with direct measurements e.g. with a laser beam as a reference line. Stochastic errors of three points instruments are in the same order or even better compared to two points instruments. They require, however, an internal reference and must be set to zero on a perfect flat reference, for example an optical flat. The propagation of zeroing errors is proportional to $\mathrm{m}^{2}$. For small lines the resulting errors are in the same order as given for the classes $3 a$ and $3 b$. For example, a zeroing error of $0.05 \mu \mathrm{m}$ (100 pitch) causes a total systematic error of $0.2 \mu \mathrm{m}$ on a $400 \mathrm{~mm}$ long line, but $5 \mu \mathrm{m}$ on a $2 \mathrm{~m}$ long line. The high systematic errors compared to the stochastic errors limits the usefulness of 3-points methods. Althuugh a need is felt for simple (preferably optical) means for precision flatness measurement of the entire surface, such methods are not available for larger surfaces. The best methods are still based on measuring lines on the surfaces, where instruments are available with sub- 
micrometer accuracy. It will be shown in the next section that a smart linking of the lines improves the overall accuracy.

\section{From straightness to flatness}

A flat is defined by two intersecting lines (Fig.2). Any additional line which has two points in common with these lines is in the same flat.

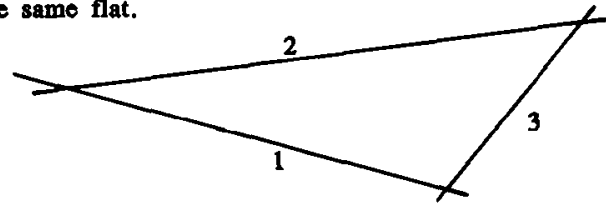

Figure 2. A flat is defined by any pair of intersecting lines.

A multi-purpose grid pattern is given in Figure 3. This grid is applied as a master grid. It can be seen as a generalization of the well-known Union-Jack pattern. For $m=n=2$ the grid equals the Union-Jack. The maximum of four measurements per grid point allows a high degree of redundancy. The grid represents the maximum

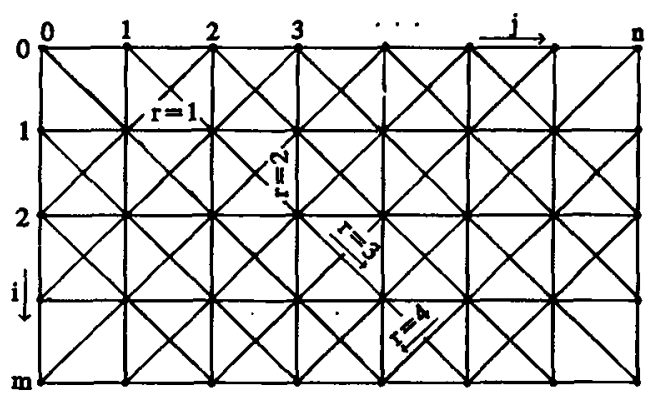

Figure 3. The base grid. Heights can be measured at the grid points $P_{v}$ with respect to each of the given lines.

number of possible measurements. Lines or points may be omitted as long as a reference plane (Fig.2) can be determined. Assuming a surface plate having a real height $H_{i j}$ in a given point $P_{i j}$, the measurements with respect to reference lines in the different directions $(r=1 . .4)$ contain orrors $\varepsilon_{\mathrm{rij}}$. Purther each reference line can be translated by a distance and tilt $\alpha$. The relation between the measurements and the beights witb respect to a reference plane is found from Fig.4:

in $j$-direction:

in i-direction:

$$
\begin{aligned}
& H_{11 j}=h_{14}-a_{1}-j \alpha_{1} \\
& H_{2 j}=h_{2 i j}-a_{2}-i \alpha_{2}
\end{aligned}
$$

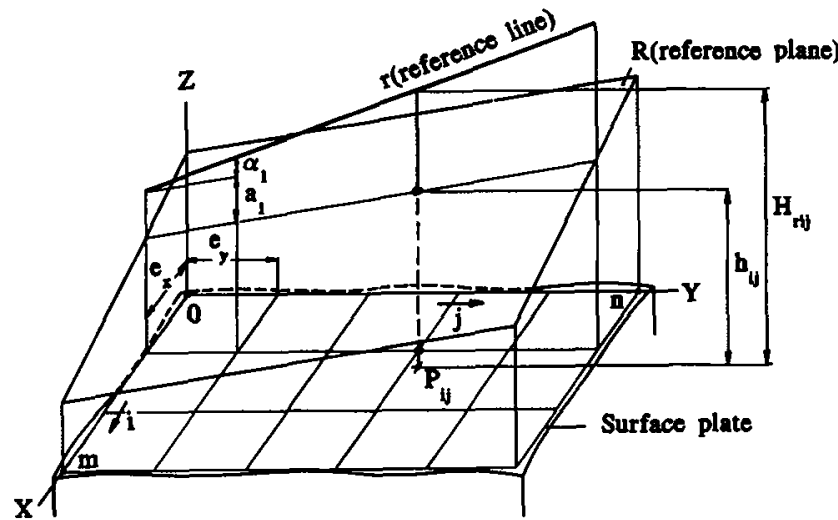

Figure 4. Coordinates, reference line and reference plane. The parameters $a$ and $\alpha$ of the line are found from the condition that the square sum of the differences between measurements (with respect to different lines in the same point) is minimum over all points simultaneously.

For the other directions of the reference lines similar equations can be derived. They can be written as:

$$
y_{1}=x_{11} p_{1}+x_{12} p_{2}+\ldots+x_{i n} p_{n}
$$

in which the vector $p$ contains all unknown h's, a's and $\alpha$ 's while the vector $y$ contains contributions of the measured $\mathrm{H}$ 's. Using the technique of multiple regression the parameters $\mathbf{P}$ follow from (Carnahan 1969):

$$
p=\left(x^{T} X\right)^{-1} x^{T} y
$$

The matrices become very large. With for example a $25 \times 25$ grid the total number of equations (2) becomes 2692 of which 965 parameters have to be solved. The number of equations was reduced drastically using the availability of two or more measurements per grid point. Then the unknown heights $h$ can be eliminated. It follows (eq.1):

$$
H_{1 i j}-H_{2 i j}=a_{2}+i \alpha_{2}-a_{1}-j \alpha_{1}
$$

Similar equations are obtained for tho other differences in the same point. In the same way as mentioned before the parameters a and $\alpha$ can bo solved from:

$$
\mathbf{p}^{\prime}=\mathbf{M}^{-1} \mathbf{y}^{\prime}
$$

A further improvement is obtained by the direct application of the least squares principle. $M$ and $y^{\prime}$ are constructed directly, point by point (only the measured points are taken into account). The number of equations become a linear function of the grid size instead of a quadratic one. For the $25 \times 25$ grid mentioned before now 289 equations with just 289 parameters (a and $\alpha$ ) are obtained. This can easily be handled by personal compaters. After solving the line parameters a and $\alpha$, the transformed heights $h_{x i j}$ are obtained from eq.1 The best approximation for the beight in a point is the mean value:

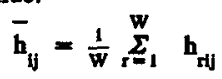

\section{Standard deviation}

In the case of direct measurements (straight edge, laser beam etc) the heights $H_{n j}$ are Normal distributed. When height differences are measured (differential mode: autocollimator, electronic level or laser-interferometer) there is a cumulation of errors along the line. However, in Fig.1 it has been shown that with the regression line as a new base the errors show an approximately bomogeneous distribution. Then in both cases a true estimate of the standard deviation of the heights $\mathrm{H}_{\mathrm{rj}}$, the "measurements", is given by eq 7 , with $F$ degrees of freedom $(F=W-N-P)$ where

$$
s_{\mathrm{m}}^{2}=\underset{1}{W} \frac{W\left(h_{\mathrm{itj}}-\bar{h}_{\mathrm{ij}}\right)^{2}}{F}
$$

\begin{tabular}{|c|c|c|c|c|}
\hline $\begin{array}{r}\text { gridsize } \\
\mathbf{m} \times \mathbf{n}\end{array}$ & $\begin{array}{c}\text { Gridpoints } \\
\text { P } \\
(m+1)(n+1)\end{array}$ & $\begin{array}{c}\text { Measurements } \\
\text { W } \\
(4 \mathrm{P}-12)\end{array}$ & $\begin{array}{l}\text { Estimated lino } \\
\text { parameters } \\
\mathbf{N}\end{array}$ & $\begin{array}{l}\text { Degrees of } \\
\text { freedom } \\
F \text {. } \\
\text { (eq. 8) }\end{array}$ \\
\hline $\begin{array}{rrr}2 & \times & 2 \\
3 & \times & 3 \\
5 & \times & 5 \\
10 & \times & 10 \\
15 & \times & 15 \\
25 & \times & 25\end{array}$ & $\begin{array}{r}99 \\
16 \\
36 \\
121 \\
256 \\
676\end{array}$ & $\begin{array}{r}24 \\
52 \\
132 \\
472 \\
1012 \\
2692\end{array}$ & $\begin{array}{r}13 \\
25 \\
49 \\
109 \\
169 \\
289\end{array}$ & $\begin{array}{r}2 \\
11 \\
47 \\
242 \\
587 \\
1727\end{array}$ \\
\hline
\end{tabular}

W the number of measurements, $P$ the number of grid points and $N$ the number of parameters. When all points are measured in accordance with Fig. 3 the number of degrees of freedom is:

$$
F_{\max }=3(m n-n-m)+2
$$

Table 1 Grid parameters.

Some numbers have been given in Table 1 . From simulations it was found that eq.7 gives an unbiased estimate indeed. From eq.1 and 6 it becomes clear that the standard deviation $\sigma_{f}$ of the results (the heights $\bar{h}_{i j}$ ) depends not only on $\sigma$ but also on the standard deviations $\sigma(a)$ and $\sigma(\alpha)$ of the line parameters, which in turn depend on the grid dimensions and the line configuration. To determine the relations between $\sigma_{m}$ and $\sigma_{\text {e }}$ Monte Carlo metbods have been applied for the line configurations shown in Fig.5. The configurations D1 and D2 can only be applied when the direction of the reference lines is known e.g. When using electronic levels. Then the parameters $\alpha$ are considered zero. As input for the simulations $N(0,1)$ distributed errors were superimposed on the data of an exact profile. After processing first the homogeneity of the output errors was checked. For all configurations the distribution of the output errors were found to be approximately homogeneous. In case D1, however, a systematic effect was found. 

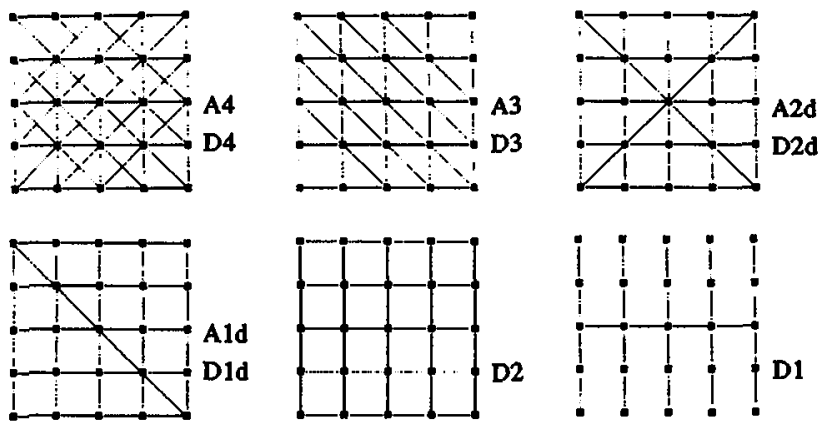

Figure 5. The ten different line configurations for which simulations have been performed. An, Dn: Absolute/Differential, in n-directions. And, Dnd: Two directions plus n-diagonals.

The relation between the output and the input errors is shown in Fig.6. In case of direct or absolute measurements (A) the accuracy increases with increasing grid size. The limit values for large grids are $V^{\frac{1}{4}}$ for $A 4, V \frac{1}{3}$ for $A 3$ and $V \frac{1}{2}$ for A1d and A2d. In case of differential measurements, however, the output errors increase with increasing gridsize. The largest output errors are found for D2 and D1 (the effect of the cumulated errors remains). For a properly chosen grid configuration the output errors will not exceed the measurement errors.

\section{Flatness parameters}

The flatness of a surface is expressed by (ISO 1990): "The distance separating two parallel planes between which that surface can just be contained". This distance is expressed by the peak to valley distance $\hat{t}$. In spite of its simplicity this definition introduces some problems: 1) The definition includes surface roughness but the measuring device, given as an example in the ISO standard, has a $\phi 10 \mathrm{~mm}$ contact area. To remove this contradiction the norm should make a clear distinction between flatness and roughness. 2) There is no calculation method available to obtain $\hat{t}$ directly from the flatness data. The problem is that no reference plane has been defined. The orientation of the reference plane depends on the planes which are looked for.

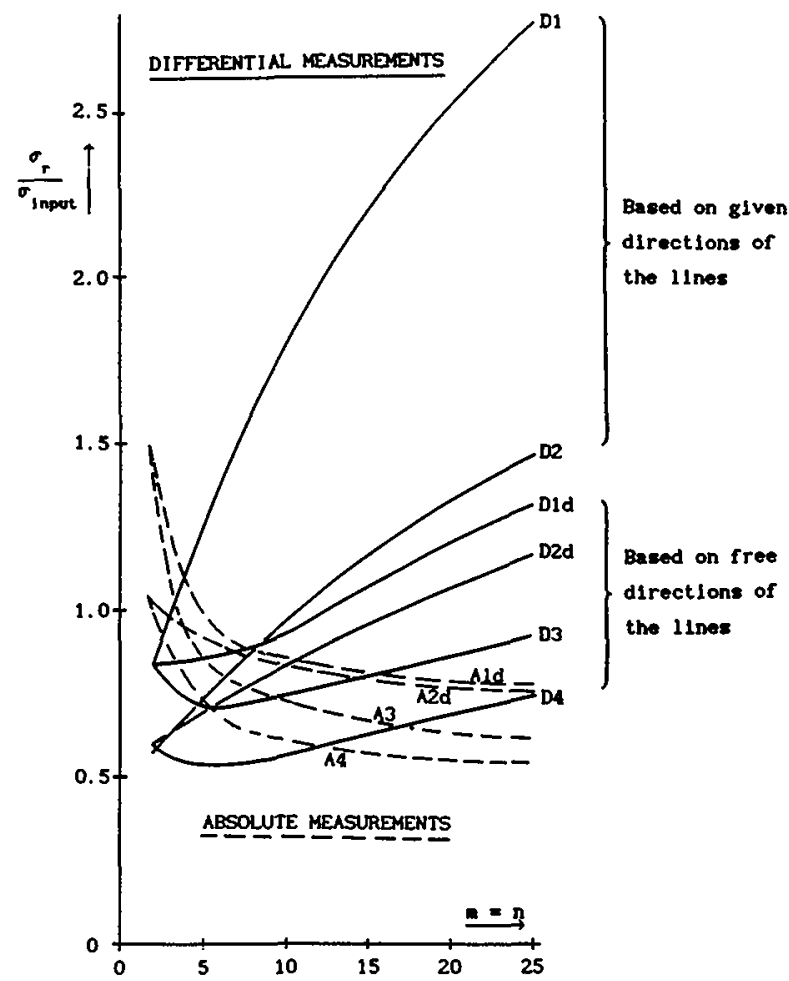

Figure 6. Relation between the output errors and the input errors for the different grid configurations.

As a consequence iterative methods must be applied. The orientation of the reference plane depends on a few points only and is sensitive to measuring errors. The flatness deviation $\hat{t}$ depends on only two points. Kampa (1978) has shown already that its value depends on the number of points measured. When measuring fewer points a lower $\hat{t}$ value will be found. A similar discussion was held in the field of surface roughness. Stout (1985) remarked: "It was soon realized that the peak to valley distance could be very misleading". Although we prefer the regression plane as the basis for flatness deviations, the minimum zone plane, following from the ISO-definition, has to be accepted as a reality.

The problem is solved in two steps. First the regression plane is taken as a basis to calculate the RMS, the peak- and the valley distance. Secondly the base plane is tilted iteratively in two directions to minimize the peak to valley distance giving the ISO parameter $\hat{t}$. Both parameters RMS and $\hat{t}$ are a measure for the quality of a surface plate do not but give information about the geometry of the surface. To that end functional parameters: sphericity, torsion and waviness have been developed to quantify the second order (sphericity and torsion) and the higher order (waviness) surface geometry. Sphericity is expressed by the bowrise b which is related to the radius of curvature by $b=l^{2} / 8 R$, where is the bow length. Torsion can also be expressed by a bowrise (Fig.7). For an easy comparison the normalized bowrises b' (per m bowlength) are preferred. Additionally the direction $\varphi$ of the torsion vector is of importance. For any direction $\theta$ the bowrise is:

$$
b_{\dot{\theta}}^{\prime}=b_{s}^{\prime}+b_{1}^{\prime} \sin 2(\varphi-\theta)
$$

From this eq. directions follow with extreme values of $b_{\theta}$, the principal axes with directions $\eta_{1}$ and $\eta_{2}$, and if $\left|b_{2}^{\prime}\right|<\left|b_{1}^{\prime}\right|$ also directions with $b_{\dot{\theta}}^{\prime}=0$ (straight lines with directions $\varphi_{1}$ and $\varphi_{2}$ ). While using the surface plate, knowledge about these directions could be very useful. Any surface can be decomposed into sphericity, torsion and remaining components. Reversely a superposition of spherical and torsion components can be made. In case of sphericity linear relations can be applied. In case of torsion the

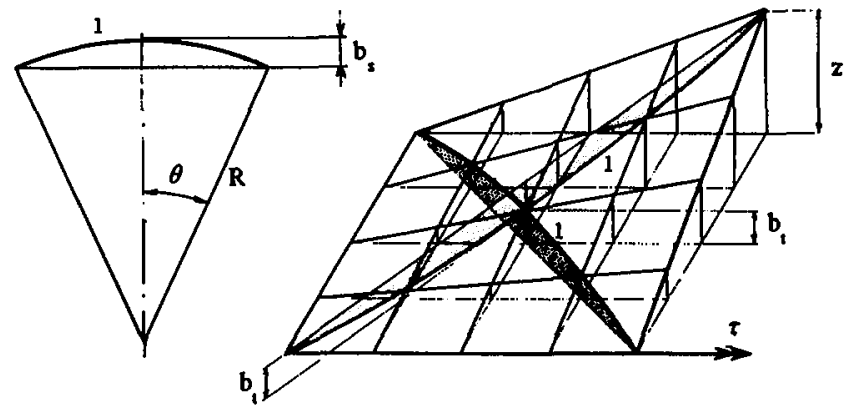

Figure 7. Sphericity and torsion can both be expressed by their bowrises $\left(b_{3}\right.$ and $\left.b\right)$.

relations are given in eq 10

$$
\left.\begin{array}{l}
\varphi_{1+2}=\frac{1}{2} \arctan \left(\frac{b_{11}^{\prime} \sin 2 \varphi_{1}+b_{12}^{\prime} \sin 2 \varphi_{2}}{b_{11}^{\prime} \cos 2 \varphi_{1}+b_{12}^{\prime} \cos 2 \varphi_{2}}\right) \\
b_{i, 1+2}^{\prime}=\frac{b_{11}^{\prime} \sin 2 \varphi_{1}+b_{12}^{\prime} \sin 2 \varphi_{2}}{\sin 2 \varphi_{1+2}}
\end{array}\right\}
$$

Sphericity is related to environmental conditions like temperatu re gradients, lightning or water contents. Abdin (1978) and De Bruin (1980) have shown the relationship unmistakably When the temperature gradient is known, the corresponding bowrise is found

$$
b_{3}=\frac{\alpha 1^{2}}{8} \frac{d T}{d z}
$$

With a given beat flux $\mathrm{L}$ through the plate (top-down), for instance due to lightning, the bowrise is:

$$
b_{s}=L \frac{1}{8}^{2} \frac{\alpha}{\lambda}
$$

For non-equilibrium situations the geometrical errors have been calculated by FEM-methods. Compared to an approximation based on top- and bottom temperature, the difference with the non equilibrium case was found to be less than $10 \%$ for a normal surface 
plate. Hence a "steady-state" temperature correction may be effective for all situations. The $\alpha / \lambda$ value is an important material property in relation to thermal errors, while the equilibrium situation will be reached faster with a higher thermal diffusivity a (Table 2). Ceramics seems the best choice. Unfortunately it is expensive and not yet available in larger dimensions.

Table 2 Surface plates materials

\begin{tabular}{|c|c|c|c|c|c|}
\hline Material & $\begin{array}{l}\alpha \\
1 / K\end{array}$ & $\begin{array}{l}\alpha / \lambda \\
\mu \mathrm{m} / \mathrm{W}\end{array}$ & $\begin{array}{c}\rho \\
\mathrm{kg} / \mathrm{m}^{3}\end{array}$ & $\begin{array}{c}\mathrm{E} / \rho \\
\mathrm{m}^{2} / \mathrm{s}^{2}\end{array}$ & $\begin{array}{l}\mathrm{a}=\lambda / \rho \mathrm{c} \\
\mathrm{m}^{2} / \mathrm{s}\end{array}$ \\
\hline $\begin{array}{l}\text { Cast Iron } \\
\text { Granite } \\
\text { Epoxy Granite } \\
\text { Ceramics }\end{array}$ & $\begin{array}{c}1110^{-6} \\
6 ! " \\
15.3 " \\
4.3 "\end{array}$ & $\begin{array}{l}0.3 \\
2 \\
8 \\
0.3\end{array}$ & $\begin{array}{l}7800 \\
2750 \\
2550 \\
3400\end{array}$ & $\begin{array}{l}1410^{6} \\
20 " \\
10 " \\
71 "\end{array}$ & $\begin{array}{l}1410^{-6} \\
1.3^{\prime \prime} \\
0.4 " \\
7\end{array}$ \\
\hline
\end{tabular}

Torsion indicates an a-symmetrical load. An example is given for a foundation plate for precision laser equipment. The first measurement was made when clamped on the machine bed and the second after releasing the clamps. The influence of the clamping was studied by taking the difference of both results as input. As shown in Table 3 the same results were obtained from eq.10.

Table 3. Influence of clamping during machining the plate.

\begin{tabular}{|lllrrrr|}
\hline & & \multicolumn{5}{c|}{ clamped released diff. eq.10 } \\
\hline Normalized sphericity & $\mathrm{b}^{\prime}$ & $\mu \mathrm{m} / \mathrm{m}^{2}$ & 12.51 & \multicolumn{1}{c|}{4.93} & 7.58 & 7.58 \\
Normalized torsion & $\mathrm{b}^{3}$ & $\mu \mathrm{m} / \mathrm{m}^{2}$ & 48.44 & 48.15 & 11.15 & 11.14 \\
Torsion direction & $\varphi^{\prime}$ & $\operatorname{deg}$ & 51.69 & 58.31 & 10.75 & 10.74 \\
Waviness & Wv $\mu \mathrm{m}$ & 10.41 & 10.46 & 0.85 & - \\
\hline
\end{tabular}

Waviness: The waviness parameter contains the higher order deviations and is expressed as an RMS-value (without sphericity and torsion). Changes of this value are associated with wear. Fig. 8 shows an example of a $1 \times 2 \mathrm{~m}^{2}$ surface plate in an automobile factory where a gully has been generated by routine measurements on the same place. The accuracy of the characteristic parameters has been obtained from the simulations too. For all configurations shown in Fig.5 the $95 \%$ boundaries have been calculated. They are expressed in units of $\sigma_{\text {m }}$ which becomes available after each measurement. For grid sizes $m$ and $n \geq 5$ the $95 \%$ boundaries for the sphericity parameter $b_{\mathrm{g}}$ have been found from $0.2 \sigma_{\mathrm{a}}$ to $0.9 \sigma_{\mathrm{a}}$

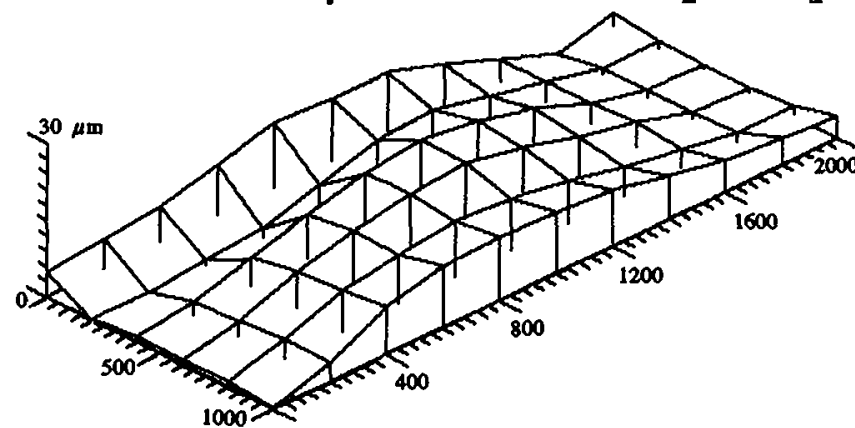

Figure 8. Typical wear pattern due to a frequent use of instruments on the same place $\left(\hat{t}=13.1 \mu \mathrm{m} \quad s_{\mathrm{m}}=0.37 \mu \mathrm{m}\right)$

depending on the grid configuration. The boundaries for the torsion parameter are approx. the double. The 95\% limits for the ISO parameter $\hat{t}$ have been obtained in the same way. Depending on the surface geometry, values between $0.8 \sigma$ and $7 \sigma$ can be expected.

\section{Instrumentation}

The method has been tested with different instruments:

1. An electronic level (Wyler), connected to an analog computer card.

2. A digital autocollimator (Moller-Wedel), connected to a serial port of the computer.

With both instruments coupled to different personal computers a plate was measured simultaneously line by line. The standard deviation was $0.13 \mu \mathrm{m}$ for the Leveltronic and $0.20 \mu \mathrm{m}$ for the Auto- collimator. No significant differences were found between the six measurements. The grid configuration was D3 (Fig.5) with 5x10 grid fields.

Another test was made with the levels only. A (nearly) perfect surface plate was measured at 2-days' intervals (grid D4, pitch $200 \mathrm{~mm}$ ). The peak to valley distance $\hat{\mathrm{t}}$ was found $0.47,0.59,0.53$ and $0.48 \mu \mathrm{m}$ respectively. The standard deviation was $0.09 \mu \mathrm{m}$. This set of measurements was also processed, taking advantage of the levels, with a fixed direction of the reference (grid D2 and D1, $\alpha=0$ ). Table 4 shows the results of one of the measurements.

Table 4. Different results from the same measurements.

\begin{tabular}{|lcllll|}
\hline & $\hat{\mathrm{t}}(\mu \mathrm{m})$ & $\sigma_{\mathrm{m}}(\mu \mathrm{m})$ & $b_{s}^{\prime}\left(\mu \mathrm{m} / \mathrm{m}^{2}\right)$ & $b_{1}^{\prime}\left(\mu \mathrm{m} / \mathrm{m}^{2}\right)$ & $\varphi\left({ }^{0}\right)$ \\
\hline 4-directions & 0.53 & 0.09 & 0.04 & -0.02 & 68.3 \\
2-directions & 0.96 & 0.27 & 0.02 & -0.11 & 8.3 \\
Difference (2-4), eq. 10: & & 0.02 & -0.12 & 4.2 \\
\hline
\end{tabular}

A significant change in torsion appears. The difference between both results, which is given in Fig.9, can be fully explained by a (constant) drift rate of the instruments as has been checked separately. When measuring in only 2 directions this drift is part of the results. In case of 3 or 4 directions all lines are supposed to be independent. Then the drift influences only the tilt angles $\alpha$ and is filtered from the results.

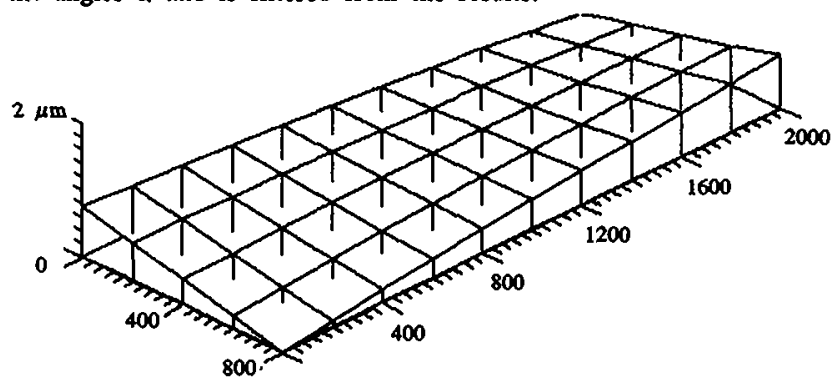

Figure 9 Drift of the instruments appears as true torsion. Conclusions

The calibration accuracy of the highest quality flat surfaces depends on the geometrical stability of the material, the environmental conditions (mainly temperature), the accuracy of the instruments and the applied measuring method. It has been shown that, with different instruments, the required shape accuracy can be obtained easily. The standard deviation is derived directly from the measurements. Dependent on the required accuracy and the instruments used, an adequate grid configuration must be chosen. The developed flatness parameters are related to functional properries of the surface. They may be used to record the history of a plate and to support decisions about maintenance.

\section{References.}

Abdin, S.Z. 1978. Investigation on Granite as a Material for Metrology Aids. Annals of the CIRP 27(1), 377-381.

Anonymus 1989. Ultra precision machining technology challenging Angstrom units. Metalworking Engng and marketing. 11(1), 31-37. de Bruin, W. and J.Meijer 1980. Analysis of Flatness Measurement and Form Stability of a Granite Surface Plate. Annals of the CIRP 29(1), 385-390.

Carnahan, B. 1969. Applied Numerical Methods. J.Wiley, New York. ISO 8512 Surface plates Part 1 and 2: In press, expected 1990.

Kampa, H. und M.Schwertz 1987. Digitale Formprtifung am Beispiel der Ebenheitsmessung, VDI-Z, 120(21), 981-987.

McKeown, P.A. 1987. The Role of Precision Engineering in Manufacturing of the Future. Annals of the CIRP 36(2), 495-501.

Melfer, J. 1989. From straightness to flatness, about surface plate measurement. Ph.D Thesis University of Twente NL, 144p. Oya, M. et al. 1987. A Study on Improvement of the Accuracy of a Three-coordinate Measuring Machine; A method of error correction. JSME Int. Journal 30(260), 344-349.

Park, C.W. et al. 1988. Forecasting compensatory control of machine flatness. Int J. Mach Tools Manufact. 28(1), 59-67.

Stout, K.J. 1985. Specification and control of surface finish: empiricism versus dogmatism. Optical Engineering 24(3),414-418. empiricism versus dogmatism. Optical Engineering 24(3),414
Taniguchi, N. 1983. Current Status in, and Future Trends of, Ultraprecision Machining and Ultrafine Materials Processing. Annals of the CIRP 32(2), 573-582.

Trumpold, H. 1988. Schlüsseltechnologien und die Qualitat techniseher Oberflacben. Feingeratetechnik 37(1), 1-6. 